en 1903, au Congrès de Médecine de Madrid, puis en 1909, à l'Académie de Médecine (Propriétés physiologiques et thér apeutiques du sérum du lait) (lacto-sérum). “ M. R. BLondel avait bien constaté certains effets physiologiques et, en particulier, la réaction hyperthermique quelquefois violente, qui suit l'injection parentérale du lait. Mais c'est surtout sur le petit lait (lacto-sérum) que portèrent ses expériences dans le but déterminé d'agir sur la tension artérielle des artério-scléreux. Les résultats qui paraissent avoir été très favorables, furent confirmés ensuite par d'autres expérimentateurs, en particulier, en France, dans les services hospitaliers des Professeurs Robin, Hughand, Vaquez et de M. Oulmont.

“ Ces injections de lacto-sérum furent essayées également par les Professeurs A. Robin et Maygrier dans quelques graves affections inflammatoires : infections puerpérales, péritonites post-opératoires, fièvre typhoïde, pneumonie. Les résultats en furent très encourageants. Il ne semble pourtant pas qu'ils aient été aussi favorables que ceux qui ont été obtenus ensuite avec le lait complet. "

Il semble donc bien que la base de cette méthode ait des assises assez solides ; l'autorité des Maîtres qui se sont, à l'origine, donnés à l'étude des injections sous-cutanées de lait et les résultats qu'ils en ont obtenus, méritent qu'on lui accorde quelque crédit. La modification, que leurs imitateurs ont apportée à la méthode : l'emploi du lait complet au lieu du petit lait, exige que les expériences thérapeutiques - d'une innocuité, on le sait, à peu près absolue - soient reprises, et que des observations précises et nombreuses nous éclairent définitivement, en France, sur la valeur de la méthode.

\title{
REVUE CRITIQUE.
}

SUR LANAPHYLAXIE LAGTEE (suite et fin) (1),

PAR

M. PÉHU,

Médecin de la Charité et

de Lyon.

\author{
P. BERTOYE, \\ Interne des Hôpitaux
}

Concurremment aux études de clinique humaine, les réactions organiques dues au lait étaient l'objet de recherches expérimentales. Le point de départ de ces travaux est la constatation faite par Anthus (1903) du pouvoir anaphylactogène du lait.

(1) Voir, Premier article, in : Le Lait, $\mathrm{n}^{\circ}$ 7, 1921. 
A dater de cette époque, de très nombreuses recherches ont été poursuivies tant en France qu'en Italie et en Allemagne. Parfois un peu confuses, elles permettent cependant de retenir un certain nombre de faits précis que nous allons envisager.

Choix de l'animal d'expérience. - L'animal le plus fréquẹmment employé pour les études sur l'anaphylaxie est le cobaye. Chez lui, en effet, on détermine avec la plus grande facilité cet état. En outre c'est un animal d'un maniment commode.

Arthus fait un reproche aux expérimentateurs de l'emploi constant de eet animal. Il lui préfère le lapin, qui présente dans ses manifestations anaphylactiques une symptomatologie beaucoup plus nuancée, se prêtant à une analyse minutieuse, à une inseription, à une mensuration qui ne peuvent être retrouvées chez le cobaye. Il reproche en outre à celui-ci une grande variabilité des conditions d'établissement, de renforcement et de conservation de l'état d'anaphylaxie. Mais, par contre, chez le lapin, l'anaphylaxie n'est pas spécifique et il ne présente pas d'antianaphylaxie.

Malgré toutes ces raisons et peut-être parce qu'il est d'un maniement plus facile, le cobaye a été préféré dans un grànd nombre de cas et c'est chez lui que beaucoup des recherches que nous rapportons ont été effectuées.

Technique des expériences. -- Lors des premières expériences, on s'aperçut très rapidement que le lait pur ne constitue pas pour le cobaye une nourriture inoflensive. Bartenstein fit remarquer avec juste raison que l'animal ne trouve pas dans cette alimentation la quantité de cellulose nécessaire au fonctionnement normal de ses voies, digestives. Dès les premiers jours, il perd du poids, présente de la diarrhée et meurt à la suite de troubes digestifs graves. Il remédia à ce défaut par l'adjonction an lait de parcelies de pain et la bouillie ainsi composée permit la survie de l'animal.

Il s'en faut toutefois que même avec un tel artifice, on réalise une alimentation parfaite et une progression régulière de la courbe de poids. Gux-Laroche, Richet fils et Saint-Girons décrivent deux types d'évolution chez le cobaye nourri de cette manière. Dans le premier, le poids moyen augmente jusqu'au $13^{\circ}$ ou $16^{\circ}$ jour et revient ensuite au poids initial. Dans le second, l'augmentation de poids ne

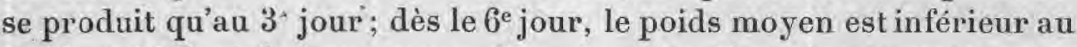
poids initial et baisse encore dans la suite. Il est à remarquer que ce sont les cobayes de ce groupe qui donnent les plus beaux résultats d'anaphylaxie.

Malgré ces troubles dans le métabolisme général des cobayes nourris au lait de vache et dont nous essaierons d'indiquer la signifi- 
eation, on peut obtenir par cette technique une sensibilisation suffisante. Le régime est à l'heure actuelle bien réglé et il faut chaque jour une ingestion de 90 à 150 grammes pour un animal de $500 \mathrm{gr}$. Cette ration doit être maintenue environ trois semaines, d'après Bartenstein. Au bout de ce temps, l'absorption d'antigène est considérée comme suffisante.

Nature de l'antigène.- Le lait de vache a été au cours des expériences le seul produit habituellement employé. BEsRedKa s'est servi du lait de chèvre, Laroche, Richet et Saint-Girons ontemployé sept fois le lait de femme, six fois le lait d'ânesse, non comme substance préparante, mais lors de l'injection déchainante, pour démontrer que la réaction n'est pas absolument spécifique pour un lait d'espèce déterminée.

En premier lieu, le lait de vache a été employé cru, et il s'est montré constamment anaphylactogène. Tous les expérimentateurs sont d'aecord à ce sujet: BEsRedka l'a prouvé d'une façon formelle ; après lui Rosenau et Anderson, Laroghe, Richet et Saint-Girons, KLeinschmidt ont confirmé ces conclusions.

Dans une seconde série d'observations, le lait a été cuit pendant un temps relativement court: 15 minutes à $56^{\circ}$ (KLEINschmidT). BESREDKa l'a porté à $120^{\circ}$; malgré cela, la recherche du choc anaphy. lactique, dans les délais normaux, a montré que l'animal réagit, d'une façon inconstante toutefois, au dire de Kueinschmidt. En dehors de cette restriction, on peut se rallier aux conclusions formulées par LAROCHE : les animaux nourris au lait cuit sont anaphylactisés comme ceux nourris au lait cru, que l'injection déchainnante soit faite avec du lait cru ou cuit.

Mais si le lait est porté à la température de $130^{\circ}$ ou $140^{\circ}$, il perd son pouvoir anaphylactogèue : cette limite a été étabiie par Besnedra. Pour expliquer pareille disparition, Dorern et Russ, Kleinschmidr invoquent le phénomène de la coagulation. Par elle serait détruit l'état physico-chimique normal, support du pouvoir anaphylactogène de l'albumine. Sous l'influence de la chaleur entre $100^{\circ}$ et $120^{\circ}$ les albumines du sérum lacté sont d'abord coagulées, et c'est ce qu explique l'inconstance des résultats produits par le lait ainsi modifié ; mais aux environs de $140^{\circ}$ la caséine est coagulée à son tour. A ce moment, l'état physico-chimique de toutes les albumines composantes du lait se trouve modifié ; le pouvoir anaphylactique disparaît.

\section{Pouvoir anaphylactogène des diverses parties com-} posantes du lait. - Le lait est composó d'une protéine spéciale, la caséine, et d'un sérum qui contient en suspension deux albumines : la lactalbumine et la lactoglobuline. En outre des graisses, un hydrate de 
carbone, le lactose, et des sels y sont également enfermés. Toutes ces substances ont été étudiées séparément.

La caséine a été incriminée tout d'abord par BEsRedKa, qui lui reconnaissait le triple pouvoir sensibilisant, vaccinant et toxique. Schricker, étudiant son effet sur un nourrisson qui présentait de l'urticaire à chaque ingestion de lait, la désigne expressément comme responsable des accidents. KLeinschmidt ne nie pas son pouvoir; il le diminue cependant pour donner la première place aux albumines du sérum lacté.

L'influence de ces dernières, en effet, a été diversement interprétée par les auteurs. BesredKa conclut: le petit lait sensibilise ou ne sensibilise pas suivant les eas, mais il est toujours vaccinant et il n'est jamais toxique. Cette opinion a trouvé dans Kleinschmidt un contradicteur énergique. Pourlui, la lactalbumine est toujours sensibilisante parfois mème en dehors de la caséine. Un choc produit avec cette dernière ne désensibilise pas pour l'albumine. Mais il peut exister une anaphylaxie polyvalente où se mêlent sensibilisation à la caséine et sensibilisation à la lactalbumine. Quant au pouvoir vaccinant du petit lait sur lequel insiste BESREDKA, puisqu'il en fait un procédé de désensibilisation au cours d'un état anaphylactique lacté, KLEINschmidt l'a nié à la suite d'une première série d'expériences. Mais dans une seconde série, il a modifié sa technique, et constaté qu'effectivement après l'absorption de petit lait, peuvent se produire encore des phénomènes graves, mais plus de choc mortel. Il lui a suffi pour cela d'inanitier le cobaye pendant $\mathbf{1 0}$ heures, ou de le purger préventivement à l'aide d'une solution alcoolique de podophyllin. Ainsi done, bien que Schricker n'ait pu reproduire chez son nourrisson, des phénomènes urticariens après l'absorption de petit lait, il semble bien découler des expériences de Kreinschmidt que les albumines du sérum lacté interviennent pour une part dans la constitution de l'état anaphylactique, et dans le déclanchement du choc.

Quant au beurre, son action est moins nette. Freund a obtenu, il est vrai, une réaction par injection de beurre. Uhlenluth et Handel. ont sensibilisé des cobayes par la même substance ; mais Kцеinsçн мiтн et Much ont montré que le beurre entraîne avec lui des albumines et qu'on ne peut dans ces conditions affirmer qu'il intervient seuldans la production du choc.

Pour résumer, il semble bien que l'action du lait soit due aux substances protéiques qu'il contient; le rôle du beurre doit être jusqu’à nouvelle expérience laissé en suspens.

Voies d'introduction du lait. - Il importe pour bien étudier celles-ci de faire le départ de l'effet que l'on veut obtenir : sensibi- 
lisant, déchainant, vaccinant. Pour obtenir l'un quelconque de ces effets, il n'est pas indifférent d'employer tel ou tel mode d'introduction; de plus le même antigène peut varier dans ses effets suivant la voie d'apport.

A. Effet préparant. - Deux voies peuvent être suivies pour obtenir cet effet : la voie entérale et là voie parentale.

La voie entérale, si elle n'est pas la première en date au cours des expériences, est la plus intéressante à étudier, car c'est elle qui se rapproche le plus des phénomènes observés en clinique et qui pour. rait nous donner une explication de ceux-ci.

BesRedKa, lorsqu'il fit en 1909, ses premières expériences, ne parvint pas à sensibiliser les cobayes par cette voie. Mais, l'année suivante, Lesne et Dreyfus, Barnathan reprirent la question de l'anaphylaxie alimentaire, et étudièrent le sort de divers antigènes dans l'estomac l'intestin grêle, le gros intestin. Bien qu'ils n'aient pas expérimenté avec du lait et qu'ainsi leurs recherches s'écartent un peu de notre sujet, nous en rapporterons rapidement les conclusions en raison de leur importance. Ils n'ont jamais obtenu d'anaphylaxie en introduisant directement de l'albumine d'ouf dans l'estomac ou l'intestin grêle; ils ont toujours eu un résultat positif en employant comme voie d'introduction le gros intestin et le rectum. Ils attribuent ces résultats non à l'acide chlorhydrique qui se montre inactif, mais aux ferments stomacaux et paneréatiques, qui transforment les substances albuminoïdes. Il est à remarquer que la transformation destoxalbuminoïdes n'est pas aussi complète et que ces mêmes ferments n'empêchent pas l'action de l'une d'elles, l'actino-congestine. C'est en se basant sur ces faits que Laroche, Rosenau et Anderson sont arrivés à sensibiliser des cobayes à l'aiłle du lait cru ou cuit ; ils ont montré que son ingestion quotidienne sensibilise le cobaye dans les conditions que nous avons indiquées lors de la description des techniques.

Il faut signaler aussi, bien qu'on ne puisse dire s'il ne s'agit pas là de phénomènes purement toxiques, le fait 1 apporté par KAssowitz d'une diarrhée, qu'il estime d'ordre anaphylactique, chez des animaux sensibilisés à la caséine par voie entérale.

Des différentes voies parentérales employées habituellement, la voie sous-cutanée semble avoir été seule ehoisie pour produire un effet sensibilisant par le lait. C'est à elle que s'est adressé Arthus dans son expérience princeps ; c'est elle qu'a employée BesREDKa, Elle s'est montrée parfaitement opérante. Elle est toutefois moins intéressante pour l'explication des phénomènes cliniques, car elle constitue une voie anormale de pénétration du lait dans l'organisme, 
Il était utile de rechercher également, si pendant la période de lactation un organisme peut se sensibiliser lui-mème. C'est ce que laissaient à penser les expériences d'Uhlentuth et Clovgr. Mais les recherches de Besredica et de Wells ont montré qu'entre le sérum et le lait de vache, de même qu'entre le sérum et le lait de femme, il n'y avait pas de relations dans le sens de l'anaphylaxie. En effer, le sérum prépare toujours mieux à l'égard d'un sérum homologue qu'à l'égard du lait homologue, et pice persa.

B. Effet déchainant. Cet effet qui est vraiment le seul critère certain de l'anaphylaxie expérimentale, il aurait été intéressant de le produire par voie digestive. Beaucoup d'auteurs s'y sont essayés : aucun n'a réussi. Il n'est à notre connaissance qu'un seul cas où le choc ait été déchaîné par ingestion seconde : c'est l'expérience de Richer qui a produit un choc par voie intestinale avec la crépitine après sensibilisation par voie parentérale. Mais il s'agit dans ce cas d'une toxalbumine, dont la composition et l'action ne sauraient être comparées à celles des albumines nutritives, du lait en particulier. Celui-ci s'est montré toujours, et sous toutes ses formes, absolument incapable de déclencher une anaphylaxie active.

C'est done à la voie parentérale qu'on s'est adressé pour faire la preuve de la sensibilisation antérieure. L'introduction du lait a été faite par voie péritonéale, par voie intra-veineuse, par voie intracardiaque (KLEINSGHMIDT), par voie cérébrale (BESREDKA). Toutes ont été suivies d'effets; mais il faut remarquer qu'on s'était assuré auparavant, que, chez des animaux non sensibilisés, l'injection de $1 / 10^{\circ}$ ou $1 / \breve{\supset}^{\mathrm{e}} \mathrm{de} \mathrm{cm}^{3}$, dose habituellement employée, n'était pas toxique pour l'animal.

Il faut signaler, à propos de l'effet déchainant, la possibilité d'apparition d'un choc cinq jours après le début d'une sensibilisation lactée par voie intestinale (Guy-LARoche). Or on ne respecte pas ainsi l'intervalle libre de $\mathbf{1 1}$ jours, observé habituellement.

G. Effet vaccinant. - Parler de cela, e'est aborder le problème de l'antianaphylaxie. Il semble bien que, pour le lait, eet effet n'ait été recherché que par voie entérale. Ciest à propos du pouvoir vaceinant du petit lait, sur lequel BESREDKA a tant insisté, que l'on a étudié son pouvoir préventif. Il s'est montré certain, mais non jusqu'à faire disparaitre complètement les phénomènes morbides. Ceux-ci sont encore graves (vomissements, diarrhée, eollapsus mème), mais le choc n'est jamais mortel alors qu'il se montre tel chez les animaux non vaccinés antérieurement.

Spécificité de l'anaphylaxie. - La question se pose de la façon suivante: un animal sensibilisé au lait de vache n'est-il sensible 
qu'à ce lait? La réponse est formelle et pour tous les expérimentateurs, elle est négative : l'anaphylaxie lactée n'est pas rigourcusement spécifique. Besredka a déterminé des chocs à l'aide du lait de chèvre, GuY-I Innoche à l'aide des laits de femme et d'ànesse. Elle n'est même pas spécifique pour les différentes parties du lait: la caséine et les albumines du sérum se montrent équivalentes.

Toxicité du lait. - Lorsque que nous avons rapporté les techniques pour la nourriture des cobayes par le lait, nous avons signalé que la courbo de poids ne présente pas la progression régulière que l'on constate chez un animal normal. Mème on la voit décroître progressivement, entraînant une cachexie de plus en plus marquée qui aboutirait à la mort, si l'on n'intervenait par un retour à l'alimentation normale. GuY-LARoche et ses collaborateurs s'étaient demandé s'il n'y avait pas lieu de rapprocher cette toxicité manifeste du lait pour le cobaye, de celle qu'il semble présenter chez certains nourrissons.

Déjà Besredra avait dit en parlant des cobayes sur lesquels il expérimentait que le lait cru avait souvent fait maigrir ces animaux et les cachectisait à la longue. Arthus avait signalé le même fait chez des animaux ayant survécu an choc anaphylactique.

Pentimalli a repris ces expériences sur le lapin, et après avoir constaté que, chez cet animal, on ne peut produire l'antianaphylaxie, il attire l'attention sur l'intoxication protéique causée par les injections longtemps prolongées de lait eru, entier ou centrifugé. Les animaux, dit-il, perdent leur vivacité, maigrissent, la peau devient rude; on voit apparaître des lésions cutanées qui aboutissent à la formalion de eroutes sanguinolentes, spécialement aux extrémitês des articulations, au museau, et aux yeux. L'animal devient cachectique.

Un pareil tableau est en tous points semblable à celui que d'autres auteurs ont obtenu, Schttenhely et Weichardt avee des protéines diverses, Segala avec de la peptone, Doerr avec des extraits d'or ganes. Tous, par ces moyens divers, ont vu se développer chez les animaux des phénomènes cachectiques.

Cet état de cachexie a une très longue durée et persiste fort longtemps après la cessation des injections de lait.

Nous venons de rapporter les principales expériences qui ont été faites au sujet de l'anaphylaxie lactée. Il nous semble que nous pouvons résumer dans les propositions suivantes les faits acquis d'une façon certaine.

$\left.1^{4}\right)$ Le lait se comporte comme un antigène. Il en a le triple pouvoir : sensibilisant, déchaînant, vaccinant. 
$\left.2^{\circ}\right)$ Le lait cru possède toutes ces propriétés. Le lait cuit jusqu'à $100^{\circ}$ se comporte de façon analogue. Ce n'est qu'à partir de $120^{\circ}$ à $130^{\circ}$ qu'il perd son pouvoir anaphylactogène.

3o) L'organisme peut être sensibilisé au lait par la voie digestive : ainsi est créée une anaphylaxie alimentaire. Il peut de même être vacciné par cette voie.

$4^{\circ}$ ) On n'a jamais pu jusqu'à l'heure actuelle produire un choc par ingestion de lait.

$\left.\breve{5}^{\circ}\right)$ Les parties composantes qui peuvent être incriminées comme cause de la réaction sont les substances protéiques: caséine et surtout albumines du sérum. Le rôle des substances grasses (beurre) n'a pu encore être élucidé.

$\left.6^{\circ}\right)$ Indépendamment de son pouvoir antigénique, le lait s'est révélé comme toxique pour l'organisme du cobaye et du lapin, jusqu'à la production d'une cachexie intense, indépendante de tout choc appréciable.

\section{VI}

Ces notions de pathologie humaine et de physiologie expérimentale étant exposées, nous désirons aborder une questios d'un ordre général. Elle se pose en ces termes : Quelle place exacte faut-il assigner aux expressions symptomatiques diverses que nous avons décrites? Depons-nous les ranger toutes dans le domaine de l'anaphylaxie ou, au contraire, n'accepter que certaines d'entre elles?

Mais, avant d'entreprendre cette discussion, il est nécessaire de résumer brièvement les notions relatives à l'anaphylaxie.

Leur fondement repose sur deux découvertes, celle de Portier et Richet d'une part, celle d'Arтues d'autre part. Schématiquement, elles peuvent être résumées de la façon suivante :

Dans l'anaphylaxie, type Portier-Richet, des substances (actino et mitylo-congestines, ete...), inoculées à des animaux, développent chez eux lors de l'injection première des actions toxiques d'intensité moyenne; mais cette introduction amène une modification organique telle que, lors d'une inoculation ultérieure, ces effe's toxiques sont considérablement amplifiés. A cotte augmentation de la sensibilité de l'organisme vis-à-vis d'un poison, Portier et Richet donnent le nom d'anaphylaxie.

La découverte de M. Artuus vise un autre ordre de faits. Ce physiologiste montre qu'unc liqueur (sérum, lait, protéines de l'œuf, etc.), faisant plus ou moins partie intégrante de l'organisme, inoculée par voie sous-cutanée ou intra-veineuse, à des animaux, ne développe tout d'abord chez eux aucunc action générale ou locale, toxique ou irritative. Mais, l'introduction réitérée de cette substance amène une 
sensibilisation de l'organisme qui, auparavant, ne possédait aucune intolérance réelle à son égard.

Telle est la différence fondamentale qui sépare les expériences de Portier et Richet, puis d'Arthus. Mais dans l'un et l'autre cas, le phénomène révélateur de la sensibilisation organique affecte des caractères qui s'imposent à l'esprit de l'observateur : c'est une manifestation brutale souvent mortelle, du moins à allures graves, alors que l'introduction de la substance étrangère n'avait tout d'abord causé qu'une action toxique modérée ou même aucune influence nocive.

Bien vite, les eliniciens comprirent combien importantes étaient, pour la pathologie, ces deux notions fondamentales : et peut-être la généralisation qu'ils tentèrent fut-elle excessive. $\mathrm{Du}$ moins, beaucoup de médecins ont réclamé pour le vaste champ de l'anaphylaxie une délimitation exacte.

A l'heure actuelle, ce champ se rétrécit. Des recherches expérimentales et cliniques ont permis de rejeter du cadre de l'anaphylaxie ce qui est " choc vasculo-sanguin », produit par une substance indifférente, incapablę̧de sensibiliser l'organisme, mais susceptible d'amener une perturbation brusque de l'équilibre humoral. Au contraire, on admet dans le groupe des états anaphylactiques tout ce qui peut s'expliquer par une sensibilisation ou par une hypersensibilisation, le choc vasculo-sanguin bien caractérisé ne constituant pas un élément indispensable de l'état anaphylactique.

En ce qui concerne le lait, la nature exacte des phénomènes par lesquels se traduit l'intolérance complète ou incomplète de l'organisme vis-à-vis de cette liqueur, prête à des discussions longues. En apparence, il n'y a aucun lien entre les signes ou symptômes constatés lors d'un essai de réalimentation lactée après une maladie digestive aiguë d'une part, et les troubles apparaissant avec soudaineté dès l'injection de lait de vache à doses infinitésimales ehez un enfant qui, à aucun moment, n'a ingéré une parcelle de cet aliment.

Au premier groupe de phénomènes, on réserve le nom d'état anaphylactique. Quant au second on le dénomme "idiosyncrasie » et on lui refuse l'accès dans le domaine de l'anaphylaxie. L'argumert invoqué est que par définition l'idiosyncrasie représente une disposition ou une constitution particulières, en vertu de laquelle un individa réagit d'une façon qui lui est propre, anormale, à l'action de certaines substances: par exemple, dans le groupe des anaphylaxies alimentaires aux oufs et au lait.

Sans doute, la définition est aisée, mais son application difficile. 
En pratique, les lignes de démarcation sont mal tranchées ; l'observation des faits cliniques et la lecture des publications médicales le démontre surabondamment.

On note parfois chez certains sujets l'ét zblissement par un mécanisme lent, d'une susceptibilité à des substances entrant habituellement dans la composition du régime alimentaire. Au cours de leur existence apparaît alors une intolérance qui, non traitée, peut subsister pendant leur vie entiêre. Cependant, on dit alors qu'il existe chez eux une idiosyncrasie acquise. Inversement des suseeptibilités dites congénitales peuvent disparaitre ; à partir d'un certain àge, une alimentation normale devient possible.

$\mathrm{Au}$ surplus, en admettant que l'idiosynerasie est congénitale. on ne résout pas le problème, on ne fait qu'en reculer la solution ; car, en dernière analyse, d'où le sujet a $t$-il, pendant la vie intra-utérine, reçu cette disposition spéciale ? De ce que, par exemple, liimmunité n'est pas acquise, mais transmise de l'ascendant au rejeton, il ne s'en suit pas qu'on la désigne sous un nom diffẻrent : c'est encore de l'immunité.

D'autre part, dans la littérature médieale, pour dénommer des états nosogragraphiques semblables, on emploie des appellations diverses. Les médecins français tendent à réduire l'anıphylaxie aux phénomènes aigus caractérisés par les manifestations bruyantes, telles que le choc, et pensent qu'il faut de toute nécessité, une sensibilisation première, acquise, de l'organisme. A l'etranger, particulièrement en Allemagne, en Autriche, en Italie, aux Etats-Unis, on incline à admettre surtout le rôle de l'hypersensibilité, qu'elle soit réalisée dès la naissance, ou qu'elle s'installe au cours de la vie. Le substratum des phénomènes est donc, aux yeux de beaucoup de médecins, représenté par une prédisposition constitutionnelle, une " diathèse \#; mais l'apparition des signes qui la décèlent est variable ; c'est pourquoi dans les publications étrangères on trouve, indifféremment employés. les termes d'état anaphylactique, d'hypersensibilité, de sensibilisation, ou d'idiosynerasie.

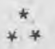

Pour exprimer toute notre pensée, nous dirons qu'on doit, $\grave{a}$ la base même de tous ces états, inooquer le phénomène d'une sensibilité accrue.

Parfois, dès le premier jour, vraisemblablement, par transmission héréditaire, l'hypersensibilité existe : e'est de l'idiosynérasie dite congénitale, appeléc d'ailleurs " anaphylaxic congénitale » par quelques mélecins, M. BARBiEr entre autres. - Ou bien c'est une sensibilisation réalisée rapidement, à la suite d'une maladie des voies diges- 
tives ; peut-être s'agit-il alors d'une anaphylaxie locale dont la muqueuse de l'estomac ou de l'intestin est le siège. Dans ces deux états le signe révélateur de la sensibilité accrue est représenté par une scène morbide à évolution aiguë, sévère ou grave comparable au choc expérimental : c'est de la grande anaphylaxie.

Par ailleurs, ce dernier plénomène manque, ou du moins, la seule observation clinique est impuissante à le déceler. Il faut alors invoquer l'action lente d'une substance perturbatrice, entrainant des modifications, probablement colloïdales du milieu intérieur : sang, cellules ou tissu, qu'elle sensibilise. C'est alors qu'apparaissent ces syndrômes dont, à l'heure actuelle, on étudie minutieusement l'étiologie : dermatoses (eczéma, urticaire, strophulus), maladies spasmodiques ayant surtout pour théâtre les voies respiratoires (asthme). Leur point commun est leur origine alimentaire ; on peut les ranger dans le groupe de la petite anaphylaxie. Ce qui, probablement, leur imprime une allure particulière, e'est que la voie d'introduction de la substance perturbatrice est représentée par le tube digestif dont l'épithélium fait subir aux substances alimentaires, particulièrement les protéiques, une transformation variable : la rapidité, l'intensité, la forme chimique ou biologique suivant lesquelles la pénétration s'effectue, dépendant de conditions dont la nature exacte nous échappe.

G'est seulement à titre d'hypothèse qu'on a incriminé l'insuffisance des sues digestifs, la perméabilité accrue de la muqueuse intestinale et l'existence de lésions hépatiques; ces dernières amenant un abaissement plus ou moins marqué de la fonction protéopexique du foie. Il est simple d'invoquer le rôle de ces facteurs, mais il est moins facile d'apporter des preuves rigoureuses de leur intervention; car, nombreuses sont les circonstances dans lesquelles ces facteurs existent sans qu'il en résulte de l'hypersensibilité ou de la sensibilisation.

\section{VII}

Dans le traitement de l'anaphylaxie lactée n'ont pas encore été réalisés de grands progrès,

Schématiquement, on peut considérer au problème différentes faces. Il s'agit d'obtenir ce que BESREDKA a appelé l'antianaphylaxie. Or celle-ci peut ètre réalisée par des moyens divers. Le plus simple consisterait, théoriquement à empêcher le nouvel apport de la substance sensibilisante, dans l'espèce : le lait de vache. Mais, pratiquement, il n'est pas toujours facile de décider cette suppression. On s'est alors ingénié à lui faire subir des modificatlons physicu-chimiques suseeptibles d'atténuer son pouvoir sensibilisant, anaphylac- 
togène. C'est dans ce but qu'ont été recommandés divers procédés de chauffage prolongé. Mais il existe à ce sujet des contradictions entre les données expérimentales et les résultats cliniques. Des recherches de laboratoire indiquent que le lait chauffé pendant 15 minutes perd son pouvoir sensibilisant; or, en pathologie humaine, les succès ne sont point réguliers ; toutetois il faut signaler que les poudres de lait, peut-être parce qu'elles sont obtenues en portant la caséine à des températures hautes, sont susceptibles de permettre une réalimentation assez rapide. On a essayé de faire pénétrer le lait par voie rectale, avec l'idée que l'introduction dans un segment d'intestin ne produit pas d'anaphylaxie ; or on sait combien l'introduction rectale est irrégulière, très rapide chez certains sujets, à peu près nulle chez d'autres. On s'est évertué à faire digérer le lait (peptonisation), pour éviter l'introduction dans le courant sanguin de produits protéiques incomplètement transformés.

Dans une autre voie on a tenté de désensibiliser le sujet par intro. duction du lait à petites doses ; et, de fait, on a réussi en procédant avec lenteur, en donnant par voie gastrique des quantités lentement eroissantes de lait de vache, administrés presque " goutte à goutte \# ou par cuillerée à café, la prudence élant alors absolument nécessaire.

Il va de soi que très souvent le moyen le plus simple, après des accidents aigus, consisterait à remplacer le lait de vache perturbateur par du lait féminin ou par un lait animal (chèvre, anesse); mais la substitution à l'allaitement artificiel du nourrissage au sein est souvent d'une réalisation très difficile à l'âge relativement éloigné où surviennent chez l'enfant les symptômes aigus de l'anaphylaxie; et c'est pourquoi l'on a tenté ces modifications chimiques de l'antigène dont nous avons parlé.

Pour désensibiliser le patient, on a préconisé des substances ne possédant aucun caractère spécifique et que, cependant, l'expérience montre efficaces, par exemple, la peplone. Les essais ne sont pas encore assez généralisés et significatifs pour qu'on puisse conclure.

D'ailleurs, tout n'est pas encore résolu dans cette question complexe de l'anaphylaxie et de l'anti-anaphylaxie lactée.Bien des points obscurs subsistent. Nous sommes encore dans une période d'attente et de recherches. L'horizon s'éclaircira sans doute lorsque nous serons en possession de méthodes basées sur des connaissances exacles touchant le mécanisme intime des états anaphylactiques. 


\section{BIBLIOGRAPHIE.}

M. Artuus, De l'anaphylaxie à l'immunité, I vol, 358 pages, ehez Masson, Paris, 1920. - M. Anthus, Lactoanaphylaxie, Société de Biologie, 1903, p. 817. - Barbier, L., Anaphylaxie pour le lait de vache chez le nourrisson, Archives de médecine des enfants, 1910, p. 499. - Barnathan, De l'anaphylaxie alimentaire Etude clinique et expérimentale, Thèse, Paris, 1910-11, Tome III. - BesnedкA, Anaphylaxie et Antianaphylaxie, Paris, Masson, 1917. - Besredks, De l'anaphylaxie lactique, Ann. de l'Institut Pasteur, 1909, p. 166.- Cassan, La protéinothérapie; ses indications et ses résultats, Rev. générale, Gazctte des Hôpitaux, 8 et 15 nov. 1919 (bibliographịe complète de la thérapeutique par les injections de lait dans les maladies toxi-infectieuses). - Castaigne et Gouraud, Anaphylaxie et intoxientions alimentaires, Journal médical français, septembre 1910. - Cnuverlorer, Procédé de vaccination subintrante de Besredka appliqué à l'anaphylaxie lactique, Société de Biolo. gie, 1911, tome $70, \mathrm{n}^{\circ} 4$. - Domec (Th.), de Dijon, Traitement d'affections oculaires diverses, par le lait de vache stérilisé en injections intra-musculaires ou hypodermiques, La clinique ophtalmologique, 1916, p. 515. Don, I., Un nouveau traitement de l'ophtalmie blennorrhagique de l'adulle, Journal de Médecine de Lyon, 20 mars 1921.- Finizio, Anafilassi familiare per il latte di muca. Tentativo di térapi antianafilactica, La Pédiatria, septembre 1911. - Finkelsfein, Le lait de vache comme cause d'accidents aigus de la nutrition chez les nourrissons, Monatschrift für Kinderheilkunde, 1905, mai, volume IV, no 2, pages 65-72, - Halberstadt, Sur lidiosyncrasie des nourrissons vis-ì-vis du lait de vache, Avchio fur Kinderheilkunde, 1911, p. 105. - Hutines, Intolérance pour le lait el anaphylaxie chéz le nourrisson, La Clinique, 10 mars 1908, p. 227. - Ноoвler, Some early symptoms suggesting protein sensitization in infancy, The american Journal of Diseases of Children, août 1916, p. 129-135, - KLeIN SCHмid, Sur lanaphylaxie Lactée, Monatschrift für Kinderheilkunde, 1913, n¹2, p. 644.-Guv-Larocae, Charles Richet Fils et SAint-Girons, Anaphylaxie alimentaire lactée. Archives de médecine expérimentale, 1911, p. 643. - J. Langle,'L'épreuve de l'hémoclasie digestive chez l'enfant, Thèse, Paris 1920-21. - Guy-Lanoche, Ch. Richet Fils et Sarnt-Grrons, L'anaphylaxie alimentaire, Actualités médicales. Chez Baillière, Paris, 1919. - I Lesné et Dreyfus, L'anaphylaxie alimentaire, Journal médical françis, 15 janvier 1913. - Lesné et Drevfus, Sur la réalité de l'anaphyliıxie par les voies digestives. Rôle de l'acide chlorhydrique, du suc gastrique et du suc pancréatique, Société de Biologie, 28 janvier 1911. - Luebliner, Rut., Choc anaphylactique grave après injection de lait, Deutsche medizinische Wochenschrift, 1918, no 20. Lust, de Bruxelles, A propos des accidents anaphylactiques provoqués par le lait de vache, Reque belge de puériculture, 25 janvier 1913. - Marfan, Traité de l'allaitement. Cliez Masson, 1920, - P. Nolf, deliège, Contribution à l'étude de la sécrétion lactée, Bulletin de l'Académie Royale de Belgique, 25 mars 1911. - PeNtimalli, Studi sull' intossicazionne proteica, IV. Toxicita 
del latte e sui derivati, La Pediatria, $1^{\text {er }}$ juillet 1921. - Pesci, Forme anafilacttiche, 1 vol., 358 p., chez Merlo et Parigi, Turin, 1916.- R honhermer, Beitrag zur Etiologie der Ucberrempfindlichkeil gegen Kuhmilch, Jalirbuch für Kinderlıtilkunde, tome 94, pages 128-130. - SAcus, Otto. Choc anaphylactique après injection du lait, Wiener klinische Wochenschrift, 1918, page 759. - Scuricker, Hans, [Contribution à l'étude de l'idiosyncrasie au lait de vache, Arch. /ür Kinderheilkunde, 1920, tome 68 4/5. - Schulz et Larson, Annpliylaxie and its relations to some diatheses commun to infancy and childhood, Arch. of ped., New-York, dée. 1919.- Tацвот, F.-B., de Boston, Idiosyncrasy to cow's milk, its relation to anaphylaxie, The Boston micdisal and Surgical Journal, 21 seplembre 1916. - E. Tenrien, Intolérance lactée et anaplıylaxie, Journal de Médccinc interne, 10 février 1911 - Weill, Ed., de Lyon, L'intolérance pour le lit chez le nourrisson et son trailement par l'injection saus-cutanée de lait, Munde Médical, juin, 1921

- Weill-Halé, L ianaphylaxie laclée, Le Nourrisson, mars 1914, page 75. - Wennstedt, Sur la question de l'jdiosyncrasie au lait de vache chez le nourrisson, Monalschrift/ür Kinderheilkunde, 1910, tome IX, page 345. - Zrberl, Idiosynerasic au lait de vache chez les nourrissons, Medizinisclie Klinik, 1910, n³0, page 1168.

\section{BIBLIOGRAPHIE ANALYTIQUE}

\section{$1^{\circ}$ LIVRES.}

\section{Wm. Feldinn. - The principles of ante-natal and post-} natal child physiology pure and applied. In- $8^{\circ}, 694$ pages, avec 6 planches, 129 figures, Longmans Green and $\mathrm{C}^{\circ}$. Ed. Paternoster-Row, London E. C. 4, 1920, 20 schillings.

Cet ouvrage est le premier de ce genre qui ait paru dans la littérature médica'e mondiale. Lauteur, assistant de Physiologie de l'Enfance a l'Hôpital des Enfants de Londres, a fait là un ouvrage très original. Dans l'introduction, il donne de suite les grandes différences qui existent entre l'adulte et l'enfant et il dirice le champ de la physiologie de l'enfance en plusieurs périodes : $1^{\circ}$ Avant le part; $2^{\circ} \mathrm{A} a$ moment de la naissance et $3^{\circ}$ après la naissance.

Dans la première, il reconnaît les stades suivants : a) germinal, b) embryonnaire et $c$ ) foetal, qui correspondent en somme à la période anté-conceptionnelle, í la conceplion et à la période post-conceptionnelle ou intrautérine.

Après le part, il distingue : le nouvenu-né, le bas-âge, c'est-à-dire la première enfance, la secunde enfance et la puberté.

On se rendra mieux comple du plan de ce travail en donnant le tilre des 41 chapitres de cet important ouvrage fort documenté : 